\title{
LAS VIRTUDES AMBIENTALES EN EL SANTUARIO PIEDRA TLACOYUNQUE, GUERRERO, MÉXICO
}

\section{THE ENVIRONMENTAL VIRTUES IN THE PIEDRA TLACOYUNQUE SANCTUARY, GUERRERO, MEXICO}

\author{
Naú Silverio Niño-Gutiérrez, ${ }^{1}$ Edmundo Enzaldo-De la Cruz ${ }^{2}$ \\ y Rosa María Velázquez-Sánchez ${ }^{3}$
}

Fecha de recepción: 25 de abril de 2017

Fecha de aceptación: 11 de diciembre de 2017

\begin{abstract}
Resumen - Piedra Tlacoyunque es mínimamente conocida a nivel nacional como santuario, de ahí que la justificación estriba en evidenciar la biodiversidad y desarrollo de este sitio. Entre los objetivos resaltan: a) sintetizar las características físico-geográficas del paisaje natural y b) destacar el uso, resiliencia y manejo sustentable actual en el lugar de estudio. El método empleado fue el enfoque geográfico, a fin de comprender de manera holista la resiliencia del sitio. Los resultados fueron: presencia de selva baja caducifolia, aguas cristalinas, mínimo oleaje, arenas de textura fina y 293 días de sol al año. Las conclusiones son: i) ostenta biodiversidad importante de ser estudiada y reconocida a nivel nacional y ii) el uso y manejo turístico actual es acertado; debido a la declaratoria como Área Natural Protegida se dedica, entre otras actividades, a la educación ambiental de residentes locales, al turismo nacional y extranjero, así como a la investigación científica.
\end{abstract}

\section{Palabras clave:}

Medio ambiente, desarrollo, biodiversidad, Tlacoyunque, santuario.
Abstract - The Tlacoyunque stone is, at least, nationally known as a sanctuary, hence the justification lies in emphasizing the biodiversity and development from this site. Among the objectives, we consider a) synthesizing the physical and geographical features of the natural landscape and b) highlighting the use, resilience and sustainable of the current management of this purpose's study. The geographical focus was the method we used to understand everything related to the resilience of the site. The results were: the characteristics of the tropical deciduous forest, crystal clear water, gentle waves, thick texture sand and 293 day-sunlight a year. The conclusions were: i) boasts important biodiversity that is nationally studied and recognized and ii) the use and current tourism management is successful because of its declaration as a Protected Natural Area which is dedicated to, among other activities, the environmental education for nearby visitors, national tourists, local people and foreigners as well as the scientific research.

\section{Keywords:}

Environment, development, biodiversity, Tlacoyunque, sanctuary.

\footnotetext{
${ }^{1}$ Departamento de Investigación, Centro de Investigación y Posgrado en Estudios Socioterritoriales (CIPES), Universidad Autónoma de Guerrero (UAGro). Correo electrónico: nausilverio@yahoo.com.mx

²Departamento de Docencia, Unidad Académica de Turismo, Universidad Autónoma de Guerrero (UAGro). Correo electrónico: zurit76@hotmail.com 


\section{Introducción}

T a naturaleza fue pródiga con el territorio nacional al dotarlo de variados paisajes, pero las actividades humanas han traído consigo el deterioro de algunos medios naturales excepcionales como macizos montañosos volcánicos, cuya materia orgánica sustenta agricultura, ganadería y en otros casos se destinan a zonas residenciales. En el estado de Guerrero dicha problemática es semejante, dado que el aprovechamiento irracional de los recursos naturales lleva al borde de la extinción a ejemplares de la selva baja caducifolia, como la caoba, parota, ceiba, entre otras.

En ese sentido, el presente artículo se compromete en un debate en favor de la conservación del medio ambiente y el desarrollo económico, mediante la declaratoria oficial de Áreas Naturales Protegidas (ANP) bajo diversas categorías federales, entre las que se incluyen: Parque Nacional, Reserva de la Biosfera, Monumento Natural, Áreas de Protección de Flora y Fauna, Parques Marinos y Santuarios, debido a que representan una opción viable para lograr la sustentabilidad local.

La Nueva Geografía Económica (NGE), cuyo máximo representante es Paul Krugman (Gasca, 2009), sostuvo desde la década de los 90 que el territorio desempeña un papel fundamental en la actividad económica de las personas que habitan las naciones, a través de su modelo centro-periferia. Este es un modelo geográfico en el que el papel central lo tiene el espacio; con dicho modelo, Krugman explicó la dinámica de cualquier porción del planeta que se encuentre poblada y en la cual se practiquen tareas económicas. Expuso también que existen dos tipos de fuerzas: las que promueven la concentración de las actividades productivas, que denominó centrípetas (Toral, 2010), y las que permiten la dispersión de las actividades económicas en el territorio, a las que nombró centrífugas (Gavira, 2010), donde el grado de aglomeración de las labores antrópicas estará en función del resultado entre el equilibrio o desequilibrio que se alcance entre ambas fuerzas.

A través de este postulado se explicarán las características físico-geográficas y manejo del santuario
Piedra Tlacoyunque, Guerrero. La importancia se manifiesta en tanto que es un estudio pionero, cuya virtud es el enfoque de la resiliencia y la sustentabilidad, perspectiva novedosa que actualmente se desarrolla en el orbe entero para entender las características propias del patrimonio natural y cultural. Dicho enfoque tiene como propósito explicar la dinámica y adaptación de los paisajes en relación con las actividades económicas que desarrolla el hombre.

La pregunta de investigación fue: ¿cuáles son los atributos ambientales actuales del santuario Piedra de Tlacoyunque? Los objetivos que se plantearon fueron: a) sintetizar las características físico-geográficas y bióticas del paisaje natural y $b$ ) resaltar el uso, resiliencia y manejo sustentable actual en el santuario Piedra Tlacoyunque.

Entre los antecedentes se encuentra que la política gubernamental conservacionista practicada en México desde la década de los 40 del siglo pasado ha promovido la declaratoria de 176 Áreas Naturales Protegidas (ANP) federales, además de las de carácter estatal, municipal y comunitario, a fin de promover la salvaguarda del patrimonio natural y contribuir al ingreso familiar al participar en la gestión de estos reservorios (Niño, 2012, p.11).

En un estudio realizado, Ruiz y Niño (2015) expusieron que la Declaratoria Oficial del Santuario fue el 29 de octubre de 1986, cuya longitud total es de 11.9 km, según la Secretaría de Medio Ambiente, Recursos Naturales y Pesca (Semarnap, 1996, p. 75); sin embargo, cabe aclarar que la porción investigada sólo cubre una longitud de 5,000 m desde la Piedra Tlacoyunque a la barra del Río San Luis, debido a que esta área es pieza fundamental de Bahías de Papanoa, de acuerdo con las ortofotos de este enclave (INEGI, 2012). La figura 1 incluye una composición de cuatro playas que presentan diversidad de paisajes, los cuales complementan las expectativas experienciales de los turistas en Costa Grande, Guerrero.

En 2016 se procedió a indagar de manera específica las características ambientales del santuario Piedra de Tlacoyunque, en San Luis La Loma, municipio de Tecpan de Galeana, Guerrero. 
Figura 1. Ubicación del santuario Piedra Tlacoyunque, Guerrero
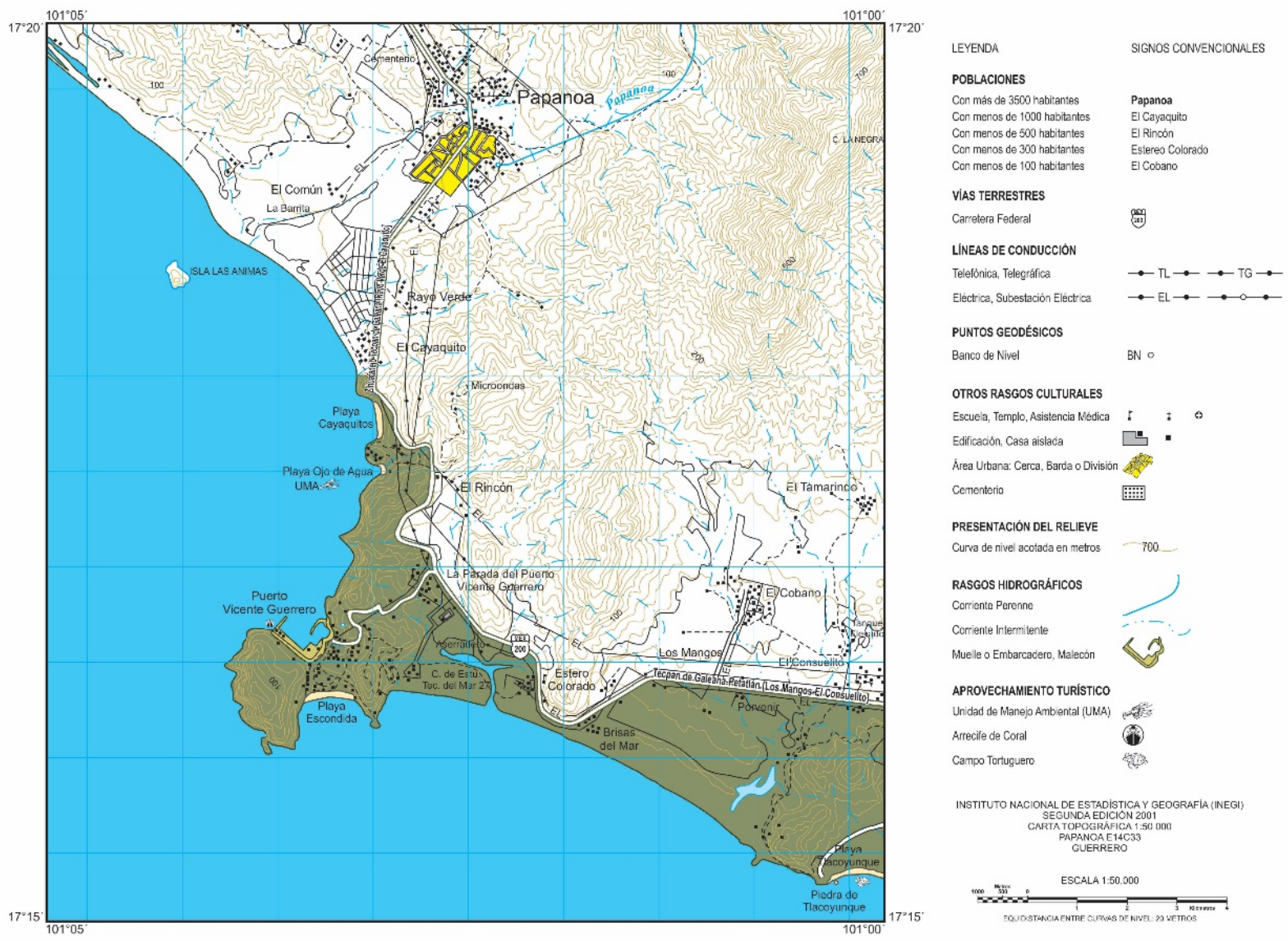

Fuente: Ruiz y Niño, 2015, p. 428.

La problemática que afecta a los estados del sur de México se vincula con la alteración del paisaje natural, asociada con la pobreza, la pérdida de recursos naturales, la contaminación ambiental, el cambio climático global y el endeudamiento económico. Con una conexión entre desarrollo sustentable y protección ambiental se tiende a proteger la salud humana, aumentar la biodiversidad, manejar ecosistemas frágiles, la agricultura sostenible, etcétera. Dicho esquema se retoma en este trabajo, dado que la conservación se asocia a la sustentabilidad cuando se practican actividades que mitigan los impactos negativos sobre el medio ambiente, para evitar el deterioro ecológico con medidas como la protección a la naturaleza y su diversidad, la contribución a un mejor nivel de vida y el abatimiento de la pobreza.
La hipótesis fue que, si han resultado positivas las estrategias y acciones fomentadas en el santuario Piedra de Tlacoyunque, entonces pueden ser retomadas en otras ANP de la misma categoría. Se emplearon publicaciones de carácter impreso sobre santuarios, Áreas Naturales Protegidas, Piedra de Tlacoyunque, así como recursos digitales en formato de CD, por ejemplo, las ortofotos de Bahías de Papanoa y su área de influencia (INEGI, 2013).

El método en que se basó el estudio fue de carácter geográfico, a fin de exponer "las características físicas y geográficas interrelacionadas con las de carácter biótico, antrópico, e incluso institucionales de cualquier enclave del orbe" (Niño, 2014, p. 18). Comprendió la lectura de materiales científicos y 
divulgativos concernientes a los temas de resiliencia, sustentabilidad, turismo de naturaleza, desarrollo, medio ambiente y geografía del turismo.

\section{Resultados}

El municipio de Tecpan de Galeana, en la Costa Grande de Guerrero, se localiza en las coordenadas geográficas extremas a $17^{\circ} 13^{\prime} 00^{\prime \prime}$ a $17^{\circ} 16^{\prime} 00^{\prime \prime}$ Latitud Norte y $100^{\circ} 56^{\prime} 00^{\prime \prime}$ a $101^{\circ} 03^{\prime} 00^{\prime \prime}$ Longitud Oeste con respecto al Meridiano de Greenwich (INEGI, 2013), que corresponde a la parte occidental del ejido de San Luis de la Loma y al ejido de Papanoa, con una pequeña propiedad entre ambos conocida como La Colonia. Se puede acceder a Piedra de Tlacoyunque a través de la carretera federal 200 Acapulco-San Luis de la Loma, en el kilómetro 150 se encuentra la desviación cuya longitud es de dos kilómetros hacia el sur para llegar al santuario.

La Piedra de Tlacoyunque tiene una altura de 35 $\mathrm{m}$ con un túnel de doble apertura y una salida que emite sonidos como resultado de la circulación de los vientos locales, por lo que se escucha como el sonar de una campana, debido a ello recibe en náhuatl el nombre de Tlacoyunque, que significa "piedra campana"; es estudiada por distintas disciplinas como la geología, litología, geomorfología y otras que comprenden factores como el clima, suelo, agua, vegetación y fauna (Niño, 2005; Niño y Saldaña, 2014). La conjunción de estos elementos da como resultado una acumulación rocosa única, cuya vía de acceso es a través de la carretera federal 200 Acapulco-Tecpan.

El lugar tiene importancia fisiográfica por ser un reservorio de biodiversidad y contar con atractivos notables, como su ubicación a sólo 20 metros de la playa local. En el medio marino, este paisaje es el pulmón verde, mismo que se aprovecha para la realización de actividades de recreo activo y pasivo, y sobre todo, educación ambiental, ya que es refugio de fauna marina, todo lo cual atrae al turismo hacia este lugar.
Desde el punto de vista geológico, la Piedra de Tlacoyunque se ubica en los límites de la Placa Norteamericana y las subplacas de Cocos-Nazca, por lo que la actividad tectónica es regular (Cetenal; 1973). Es precisamente el municipio de Tecpan un área de subducción; en ella, la placa continental se introduce hacia la placa oceánica, razón por la cual es una zona sísmica muy activa.

La Piedra de Tlacoyunque está conformada por rocas ígneas intrusivas, integradas por gneis y esquistos (INEGI, 2004), en general son difíciles de erosionar aun ante la presencia de oleaje fuerte. La zona de contacto mar-tierra se compone de afloramientos graníticos en forma de única acumulación rocosa. Esta área coincide con Zona Federal Marítimo-Terrestre (Zofemat), que va de 0 a 20 msnm en la isla y que según la Ley Número 137 de Turismo del estado de Guerrero y su reglamento, puede ser considerada de desarrollo turístico prioritario en función de sus características naturales y ecológicas, que constituyen un atractivo turístico que coadyuva al desarrollo endógeno del municipio de Tecpan de Galeana.

La geomorfología tiene que ver con las diversas formas del terreno, como las elevaciones seguidas de laderas cerriles diminutas y planicie aluvial. Las pendientes en el macizo rebasan los $45^{\circ}$. Desde la perspectiva geomorfológica, el macizo se ubica en la planicie aluvial de la Llanura Costera del Océano Pacífico (INEGI, 2005).

El clima es cálido subhúmedo con lluvias en verano (Aw1), época en que caen lluvias torrenciales entre los meses de mayo a noviembre, las cuales aportan agua suficiente para las tareas productivas y domésticas. Las precipitaciones medias anuales son de $1415 \mathrm{~mm}$, según datos del año 2013, que fue el más lluvioso en décadas, con las tormentas "Manuel" e "Ingrid", que causaron grandes daños. El promedio de días nublados es de 72 al año. La oscilación térmica es de $0.6^{\circ} \mathrm{C}$, entre $26.5^{\circ} \mathrm{C}$ y $27.1^{\circ} \mathrm{C}$. La temperatura media anual es de $26.5^{\circ} \mathrm{C}$, "donde la presencia del mar juega un papel importante como regulador térmico local" (Vidal, 2005, p. 173). 
Durante el verano, el continente registra mayor temperatura y menor presión, en tanto que el océano menor temperatura y mayor presión, debido a ello el viento se desplaza del mar hacia tierra firme con humedad alta (de hasta $80 \%$ ), en tanto que en invierno los vientos dominantes van de norte a sur, lo cual coincide con el registro de las temperaturas más bajas, según la Primera Ley de Buys-Ballot que expresa: "los vientos se desplazan de la alta a la baja presión" (Fabián y Escobar, 2002, p. 241).

El suelo Litosol se expresa a través de afloramientos rocosos, ligeramente ácidos, bajo contenido de materia orgánica y capacidad agrícola nula, por lo que su vocación natural es para vida silvestre, ya que sustenta vegetación con mínimas necesidades edáficas, aunque también se reconocen los suelos aluviales y Regosol eútrico, además de Cambisol eútrico, Phaeozem háplico, con buena proporción de materia orgánica, aunque poco poroso, limitada permeabilidad y escasamente drenado (SPP, 1981).

Las asociaciones de suelos son dos, en orden de mayor distribución espacial aparece la que se encuentra conformada por Feozem háplico-Cambisol eútrico, seguido de la asociación Regosol eútricoLitosol, lo cual establece la potencialidad de la zona para vida silvestre, como sucede en los terrenos altos y relativamente bajos. La vocación natural de la planicie aluvial funciona como vaso colector de escurrimientos fluviales, con desarrollo de vegetación riparia y crecimiento de pastos halófilos (Cetenal, 1973).

La vegetación es predominantemente nativa, que comprende a la selva baja subperennifolia y el estrato inferior integrado por vegetación natural compuesto por pastizal (1.67\% de la superficie municipal), zacate (Aristida sp); vegetación secundaria arbórea $(0.95 \%$ de la superficie municipal), tepezcohuite (Quercus conspersa), amate (Ficus sp.); selva baja caducifolia con vegetación secundaria arbustiva (52.91\%), en peligro de extinción cóbano (Sweetania sp.); vegetación de manglar $(0.28 \%)$, mangle botoncillo (Conocarpus erecta L) (INEGI, 2004). Ejemplares de frutales tales como limón dulce y cajeles (Citrus $x$ alimetta), cuajinicuil (Ingo sporia), coacoyul (Cryosophila nana), cayaco (Elaeis oleifera), ciruela (Prunos casasus, P. doméstica), almendro (Punica granatum), huamúchil (Pithecellobium dulce) y otros.

En los poblados de San Luis La Loma, San Pedro, Cóbano, Consuelito y La Colonia se observan árboles de frutos comestibles como el mango (Mangífera índica L.), ciruela criolla (Spondias mombín L.), nanche (Byrsonima crassifolia L.), chicozapote (Manilkhara zapota L.), marañona (Anacardium occidentale L.), tamarindo (Tamarindus índica L.), palma de coco (Cocos nucífera L.), papaya (Carica papaya L.) y almendro (Terminalia catapa L.) (Bolívar, Valencia y Sauri, 2009, pp. 52-61). Durante la época húmeda se observa un follaje denso y verde, y durante el estiaje se pierde el follaje y entra al estado latente, lo cual permite observar un paisaje conservado con equilibrio natural que funge como pulmón local. En éste:

Hay batracios en peligro de extinción como la rana (Rana montezumae B.), sapo (Bufo compactilis W.), incluso ajolote (Ambystoma ordinarium), además de la iguana negra (Ctenosaura pectinata) y la boa constrictor (Boa constrictor) (Gil y Castillo, 2005, p. 200).

Entre otros mamíferos, se encuentran tuzas (Geomys heterodus) y aves como el búho real (Bubo virginianus), zopilote rey (Sarcoramphus papa), calandria de agua (Icterus cucullatus), urraca hermosa cara blanca (Calocitta formosa) y jilguero (Myadestes obscurus) o incluso aves marinas como pelícanos (Pelecanus erytrorhinchus), patos pichiches (Dendroygna autumnalis) y garzas de mar pescadoras (Egreta alba) (Araujo, 2003, pp. 37-46).

La fauna de carácter marino comprende tortugas marinas laúd (Dermochelys coriacea), golfina (Lepidochelys olivacea); peces como el robalo (Centropomus originalis), pez vela (Istiophorus platypterus), dorado (Salminus maxillosus), jurel (Trachurus picturatus), sierra (Thyrsitis atun), 
huachinango (Lutjanus campechanus), pez agujón (Tylosurus acus), mero (Epinephelus sp.), pez lora (Scaropus sp.), pez globo (Arotron sp.) y mantarrayas o Raya (Urolophus sp.) (Araujo, 2003, pp. 97-101).

Existe gran diversidad de especies marinas en el área de influencia de la Piedra de Tlacoyunque, debido al elevado número de arrecifes rocosos y los peces de la plataforma continental del Pacífico Central, en su mayoría, pertenecen a la confluencia de fauna panámica y Golfo de Baja California. El área en estudio, en particular, "se encuentra en una zona de transición de las corrientes de California y la contracorriente ecuatorial del sur" (Palacios, 2002, p. 47). La biodiversidad marina se refugia en los acantilados rocosos, donde hay elevada presencia de cuevas y grietas, así como contacto con el sustrato arenoso. Por todo ello, la Piedra de Tlacoyunque es un santuario definido como:

áreas protegidas caracterizadas por una considerable riqueza de flora o fauna por la presencia de especies o subespecies o hábitats de distribución restringida. Abarcan cañadas, vegas, relictos, grutas, cavernas, cenotes, caletas u otras unidades topográficas o geográficas que requieren ser preservadas o protegidas (Semarnap, 1996, p. 19).

En el caso de la Piedra Tlacoyunque, como se encuentra a la orilla del mar, es considerada de categoría federal y sólo incluye una franja de $20 \mathrm{~m}$ desde la pleamar (INEGI, 2007), en la cual se debe evitar hacer construcciones, a menos que se considere edificar algunas en beneficio propiamente del sitio, o por otras causas de importancia superior que sean estrictamente necesarias.

La población asentada en los poblados vecinos de Cóbano, Consuelito, San Francisco, Rancho Alegre del Llano, San Pedro, San Luis de La Loma, San Luis San Pedro, Tecpan y La Colonia es de 25 mil habitantes (INEGI, 2015), algunos de los cuales prestan servicios turísticos a quienes admiran la naturaleza, por lo que el turismo es una actividad económica redituable en los municipios costeros mexicanos, incluido el correspondiente a Tecpan, Guerrero. Cada vez que llegan autobuses de visitantes (80 personas al día), el uso del suelo es predominantemente restaurantero, recreativo intensivo en la playa y recreativo pasivo en el macizo rocoso.

Es importante citar que las actividades turísticas practicadas y la educación ambiental in situ son planificadas, porque se desarrollan en relativa armonía con el medio a fin de que se sostengan en el tiempo. Conviene recordar que los mayores impactos del turismo sobre el entorno natural se observan en los ecosistemas costeros, como consecuencia del desarrollo masivo del "turismo de sol y playa, y de las actividades turísticas y recreacionales con él asociadas, y que representa $85 \%$ del turismo mundial y mantiene una industria de 385 mil millones de dólares" (Acerenza, 2006, p. 72).

El turismo que llega a la Piedra Tlacoyunque todavía no es una amenaza, ya que la mayor parte se queda en las playas cercanas, por lo que la afluencia hacia la selva es mínima y se reduce a profesionistas, estudiantes, académicos, etcétera. A la fecha no ponen en riesgo la conservación de este enclave, la cual se apega a "La Carta de la Tierra, que tiene entre otros principios respetar la Tierra y la vida en toda su diversidad; impulsar el estudio de la sostenibilidad ecológica y promover el intercambio y la extensa aplicación del conocimiento adquirido"(Chávez, 2005, p. 83), fortalecer a las comunidades locales habilitándolas para que puedan cuidar sus propios ambientes y asignar la responsabilidad ambiental en aquellos niveles de gobierno en que pueda llevarse a cabo de manera efectiva, entre otros.

Es necesario subrayar que en este santuario:

se encuentra el campamento tortuguero más grande del estado de Guerrero; en él se liberan aproximadamente cien mil individuos por año, de tres especies de tortugas: un $70 \%$ es la golfina (Lepidochelys olivacea), un $20 \%$ laúd (Dermochelys coriacea) y un $10 \%$ tortuga prieta (Chelonia agassizii) (Ruiz y Niño, 2014, p. 21). 
El turismo ha favorecido a los emprendedores, quienes gracias a su creatividad promueven La Piedra de Tlacoyunque, lo que les ha permitido mejorar su economía familiar, además de preservar los ecosistemas terrestres y marinos al asumir con responsabilidad los principios de sustentabilidad (figura 2).

Figura 2. Piedra de Tlacoyunque

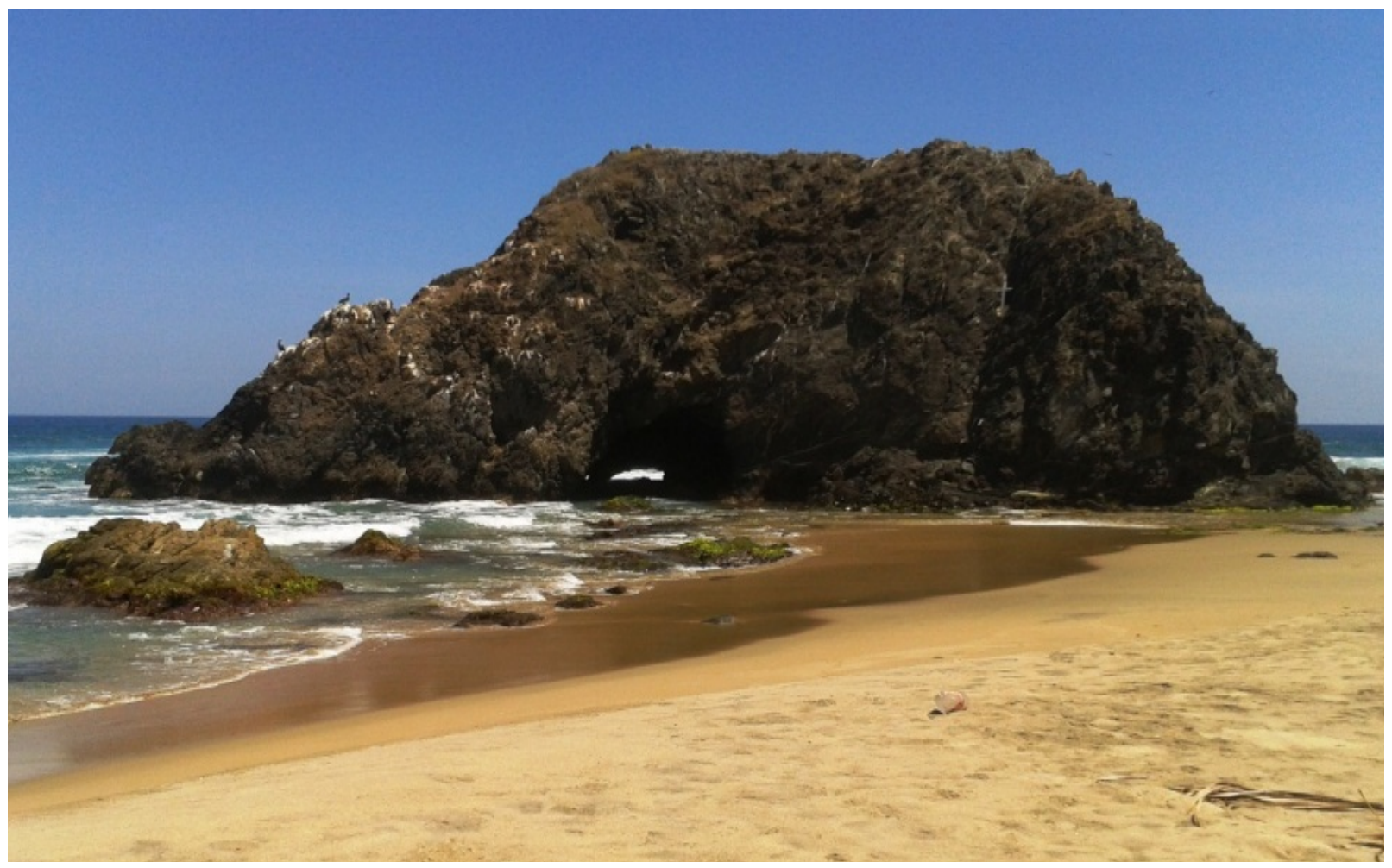

Fuente: Enzaldo, 2016

\section{Conclusiones}

La metodología aplicada permitió alcanzar satisfactoriamente los objetivos planteados mediante la identificación de la Piedra Tlacoyunque como santuario que a la fecha es resiliente; se practica turismo de naturaleza acorde con la sustentabilidad local, donde se especificaron las virtudes del enclave a través de variables geológicas e incluso del sustrato litológico, la morfología del terreno y suelos, la red fluvial, las formaciones vegetales, así como su abundancia florística, la fauna silvestre, las condiciones climáticas y los rasgos de protección de la vegetación de selva baja caducifolia. Estos atributos forman parte de la riqueza paisajística local, que es un atractivo más para los visitantes nacionales y extranjeros que arriban de manera organizada. 


\section{Referencias}

Acerenza, M. (2006). Efectos económicos, socioculturales y ambientales del turismo. México: Trillas.

Araujo, J. (2003). Fauna Silvestre del Estado de Guerrero. México: Guerrero Cultural Siglo XXI.

Bolívar, N., Valencia, M. y Sauri, E. (2009). Recursos fitogenéticos de origen tropical. Su contenido de micronutrientes. México: Universidad Autónoma de Campeche.

Cetenal. (1973). Carta Edafológica. Escala 1:50 000, México: INEGI.

Chávez, J. (2005). Ecoturismo TAP. Metodología para un turismo ambientalmente planificado. México: Trillas.

Enzaldo, E. (2016). Álbum fotográfico personal resultante del trabajo de campo realizado en 2016. Inédito.

Fabián, E. y Escobar, A. (2002). Geografía General. México: Mc Graw-Hill.

Gasca, J. (2009). Geografía Regional. La región, la regionalización y el desarrollo regional en México. México: Instituto de Geografía de la Universidad Nacional Autónoma de México.

Gavira R., M. A. (2010). Apuntes de economía regional. Pereira, Colombia: Universidad Católica Popular del Risaralda. Recuperado de http://biblioteca.utec.edu. sv/siab/virtual/elibros_internet/55767.pdf

Gil, J. y Castillo, B. (2005). Desplazamiento de vertebrados terrestres como impacto del desarrollo urbano-turístico en una selva baja caducifolia al sureste de la bahía de Puerto Marqués, Municipio de Acapulco, Guerrero, México. En R. Wences, L. Sampedro, R. López y J. Rosas (coords.), Problemática territorial y ambiental en el desarrollo regional (pp. 183-203). México: AMECIDER/UCDR/UAGro.

Instituto Nacional de Estadística y Geografía (INEGI). (2004). Sistema para la consulta del Cuaderno estadístico municipal. Acapulco de Juárez, Guerrero 2004. México: autor.

INEGI. (2005). Aerofotografía. Escala 1:75 000. G1410. México: autor.

INEGI. (2007). Aerofotografía. Escala 1:40 000. G1410. México: autor.

INEGI. (2012). Anuario estadístico del estado de Guerrero. México: autor.

INEGI. (2013). Ortofotos de las bahías de Papanoa y su área de influencia. Escala 1:20 000. México: autor.

INEGI. (2014). Cuaderno Estadístico del Municipio de Técpan de Galeana 2014. México. autor.

INEGI. (2015). Anuario estadístico del estado de Guerrero. México: autor.

Niño, N. (2005). Propuesta de manejo para lograr la sustentabilidad económica, social y ecológica en el Área Natural Protegida "Región Volcánica" del Valle de Santiago, Guanajuato. En VII Congreso Nacional y I Internacional de Investigación Turística. México: SECTUR-CESTUR-Departamento de Turismo, Recreación y Servicios de la U. de G.

Niño, N. (2012). Ecogeografía y sustentabilidad en la isla Roqueta de Acapulco. México: Editorial Praxis.

Niño, N. (2014). Planeación aplicada al caso de La Roqueta. México: Ediciones Eón. 
Niño, N. y Saldaña, J. (2014). Potencialidad del cerro La Batea para la práctica del geoturismo En N. Niño y J. Saldaña (coords.), Comunicación social del desarrollo y biodiversidad (pp. 25-31). México: Ediciones Eón.

Palacios, D. (2002). Abundancia y diversidad de peces de los arrecifes rocosos de la región de Acapulco, Guerrero. (Tesis de Licenciatura). Escuela Superior de Ecología Marina, Universidad Autónoma de Guerrero, México.

Ruiz, J. y Niño, N. (2014). El turismo en las bahías de Papanoa. En N. Niño, R. Bergerety A. Díaz (coords.), Gestión Sustentable del Turismo (pp. 17-25). México: Editorial Praxis-Conacyt-Universidad Autónoma de Guerrero.

Ruiz, J. y Niño, N. (2015). Territorio y potencial turístico en Bahías de Papanoa. Revista Mexicana de Ciencias Agrícolas, 1(12), 427-433.
Secretaría de Medio Ambiente, Recursos Naturales y Pesca (Semarnap). (1996). Programa de áreas naturales protegidas de México 1995-2000. México: autor/Instituto Nacional de Ecología.

SPP. (1981). Carta edafológica Acapulco E14-11. Escala 1: 250 000. México: INEGI.

Toral, M. (2010). El factor espacial en la convergencia de las regiones de la Unión Europea (19801996). (Tesis de Doctorado). Facultad de Ciencias Económicas y Empresariales de la Universidad Pontificia Comillas de Madrid, España. Recuperado de http://www.eumed.net/tesis-doctorales/ata/ index.htm

Vidal, R. (2005). Las regiones climáticas de México. México: Instituto de Geografía de la Universidad Nacional Autónoma de México.

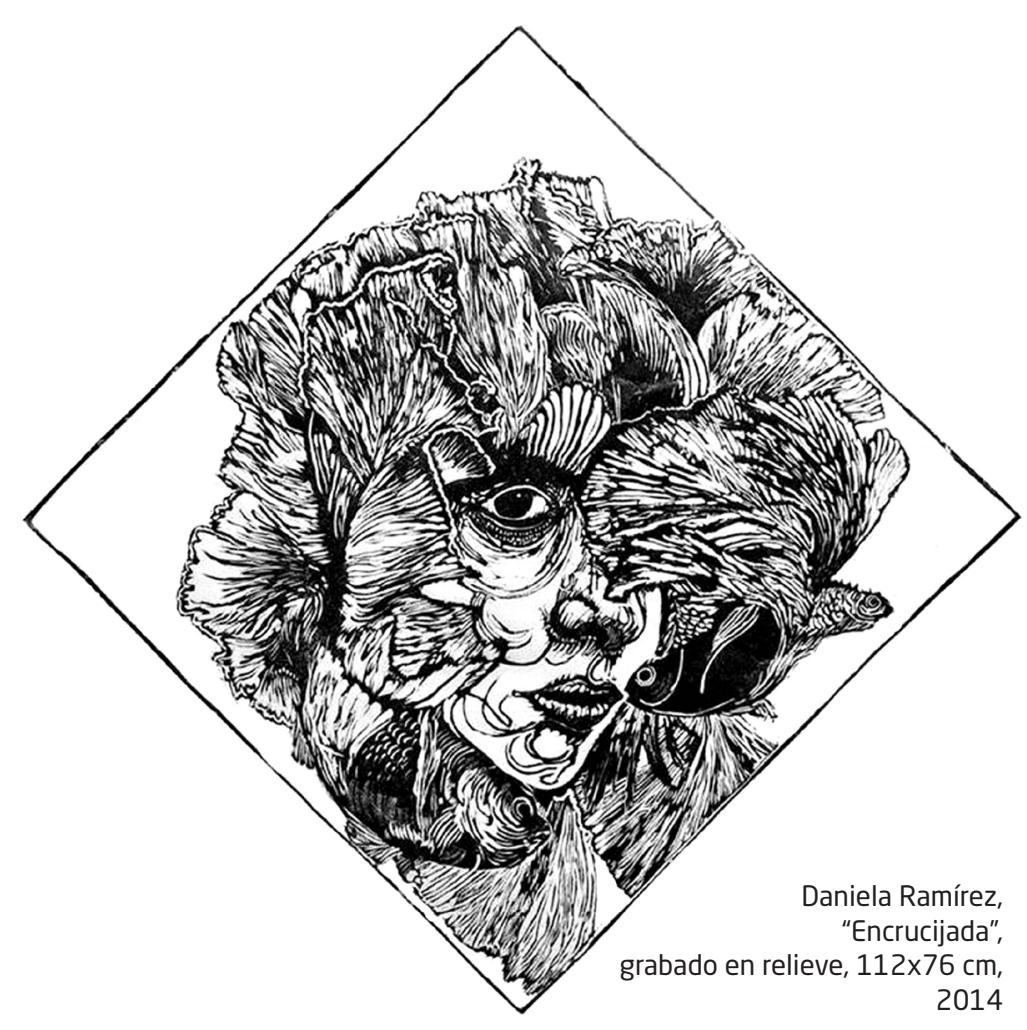




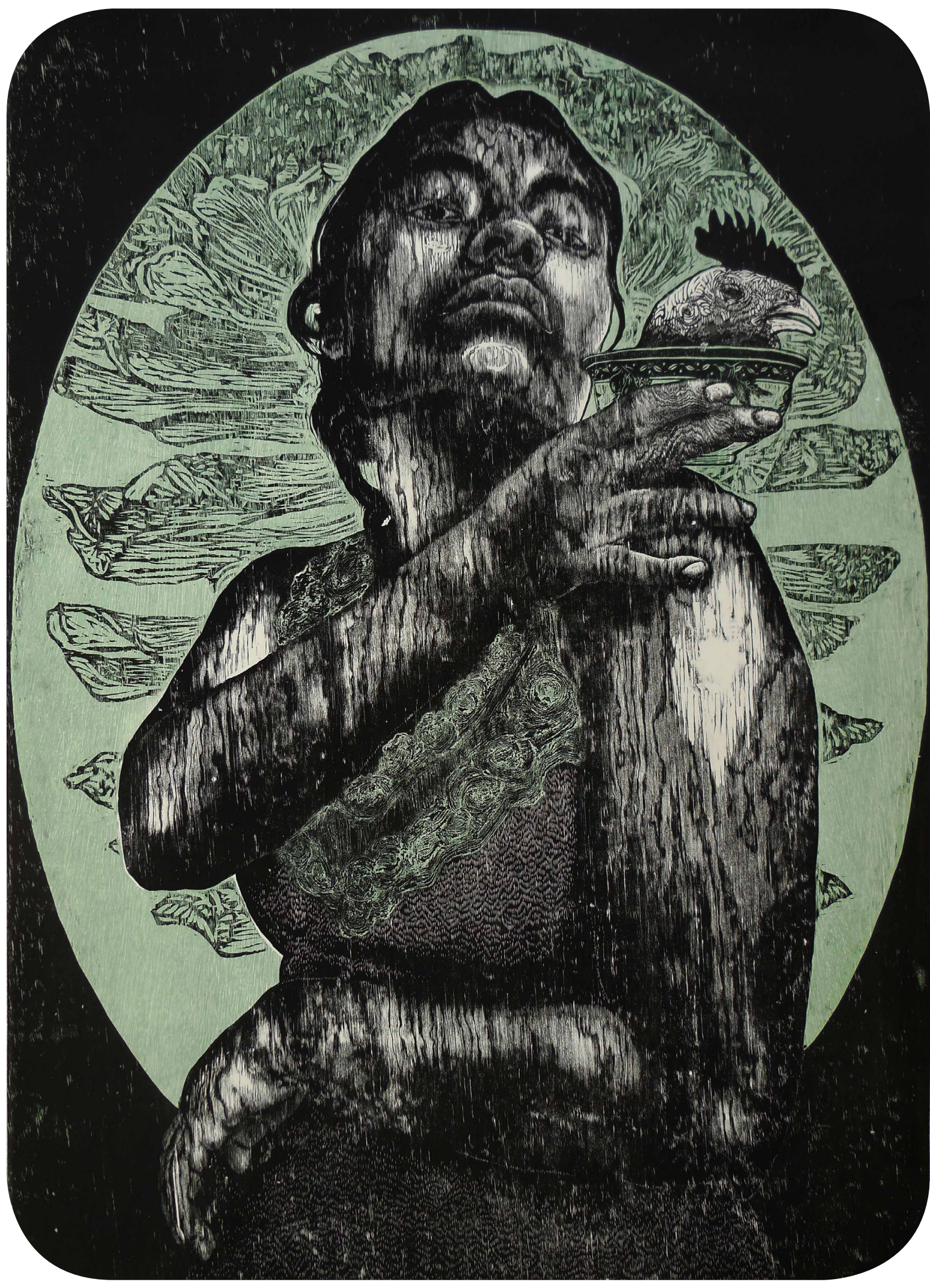

Edith Chávez 